\title{
Qualidade de vida de cuidadores de idosos: uma revisão de literatura
}

\author{
Quality of life for elderly caregivers: a literature review \\ Calidad de vida para los cuidadores de ancianos: revisión de la literatura
}

Recebido: 09/11/2021 | Revisado: 15/11/2021 | Aceito: 16/11/2021 | Publicado: 26/11/2021

\author{
Anna Flávia Almeida Macedo \\ ORCID: https://orcid.org/0000-0002-4283-2388 \\ Centro Universitário de Patos de Minas, Brasil \\ E-mail: annafalmeida@yahoo.com \\ Ana Carolina Ramalho dos Reis \\ ORCID: https://orcid.org/0000-0001-7346-842X \\ Centro Universitário de Patos de Minas, Brasil \\ E-mail: carolramalhor@gmail.com \\ Isabella Medeiros de Lima \\ ORCID: https://orcid.org/0000-0001-5406-2686 \\ Centro Universitário FIPMOC, Brasil \\ E-mail: bella1303@gmail.com \\ Maria Tereza Teixeira de Almeida Gonçalves \\ ORCID: https://orcid.org/0000-0003-4941-5605 \\ Faculdade de Minas BH, Brasil \\ E-mail: mariaterezaalmeidag@ hotmail.com \\ Kelly Vargas Londe Ribeiro de Almeida \\ ORCID: https://orcid.org/0000-0001-9252-559X \\ Centro Universitário de Patos de Minas, Brasil \\ E-mail: kellylonde@unipam.edu.br
}

\begin{abstract}
Resumo
Introdução: $\mathrm{O}$ envelhecimento populacional vem ganhando bastante destaque nos últimos anos devido ao aumento da expectativa de vida e modernização das tecnologias. De acordo com o Ministério da Saúde, o Brasil possui a quinta maior população idosa do mundo, com cerca de 28 milhões de pessoas com 60 anos ou mais. Objetivo: analisar as evidências através de uma revisão de literatura sobre a qualidade de vida dos cuidadores de idosos, identificando as principais comorbidades entre eles e a faixa etária. Metodologia: Trata-se de uma revisão de literatura nas bases de dados Lilacs, Google Acadêmico, Scientific Electronic Library Online - SciELO, US National Library of Medicine Nacional Institutes of Health (PubMed), Index Psicologia e Coleciona SUS, no período entre 2010 a 2021. Foram analisados 18 artigos. Resultado e discussão: Identificou-se que os principais fatores que influenciavam para a qualidade de vida variavam entre a sobrecarga e ao vinculo criado entre o cuidador e o idoso. Também, as principais comorbidades encontradas entre os cuidadores são: doenças cardiovasculares, psiquiátricas e musculoesqueléticas. Conclusão: Nota-se a necessidade do olhar mais cuidadoso diante da qualidade de vida dos cuidadores de idosos devido ao prejuízo à saúde destes, seja física ou emocional. Capacitando os profissionais de saúde na realização de estratégias de intervenção, a fim de criar vínculos terapêuticos com as famílias dos idosos, identificando as suas necessidades, e assim, diminuir o estresse e sobrecarga.
\end{abstract}

Palavras-chave: Cuidadores; Envelhecimento; Qualidade de vida; Saúde do idoso.

\begin{abstract}
Introduction: Population aging has gained considerable prominence in recent years due to increased life expectancy and modernization of technologies. According to the Ministry of Health, Brazil has the fifth largest elderly population in the world, with about 28 million people aged 60 years and over. Objective: to analyze the evidence through a literature review on the quality of life of caregivers of the elderly, identifying the main comorbidities between them and their age group. Methodology: This is a literature review using the Lilacs, Academic Google, Scientific Electronic Library Online - SciELO, US National Library of Medicine Nacional Institutes of Health (PubMed), Index Psicologia and Coleciona SUS data bases, from 2010 to 2021. 18 articles were analyzed. Results and discussion: It was identified that the main factors that influenced the quality of life varied between the burden and the bond created between the caregiver and the elderly. Also, the main comorbidities found among caregivers are: cardiovascular, psychiatric and musculoskeletal diseases. Conclusion: There is a need for a more careful look at the quality of life of caregivers of the elderly due to the damage to their health, whether physical or emotional. Enabling health professionals to carry out intervention strategies in order to create therapeutic bonds with the families of the elderly, identifying their needs, and thus, reducing stress and burden.
\end{abstract}

Keywords: Caregivers; Aging; Quality of life; Elderly health. 


\section{Resumen}

Introducción: El envejecimiento de la población ha ganado un protagonismo considerable en los últimos años debido al aumento de la esperanza de vida y la modernización de las tecnologías. Según el Ministerio de Salud, Brasil tiene la quinta población de ancianos más grande del mundo, con alrededor de 28 millones de personas de 60 años o más. Objetivo: analizar la evidencia a través de una revisión de la literatura sobre la calidad de vida de los cuidadores de ancianos, identificando las principales comorbilidades entre ellos y su grupo de edad. Metodología: Se trata de una revisión de la literatura en las bases de Lilacs, Google Académico, Scientific Electronic Library Online - SciELO, US National Library of Medicine Nacional Institutes of Health (PubMed), Index Psychology y Collects SUS, de 2010 a 2021. Se analizaron 18 artículos. Resultados y discusión: Se identificó que los principales factores que influyeron en la calidad de vida variaron entre la sobrecarga y el vínculo creado entre el cuidador y el anciano. Asimismo, las principales comorbilidades encontradas entre los cuidadores son: enfermedades cardiovasculares, psiquiátricas y musculoesqueléticas. Conclusión: Existe la necesidad de una mirada más atenta a la calidad de vida de los cuidadores de ancianos por el daño a su salud, ya sea física o emocional. Permitir que los profesionales de la salud realicen intervenciones con el fin de crear vínculos terapéuticos con las familias de las personas mayores, identificando sus necesidades y, así, reduciendo el estrés y la carga.

Palabras clave: Cuidadores; Envejecimiento; Calidad de vida; Salud de los ancianos.

\section{Introdução}

O envelhecimento populacional vem ganhando bastante destaque nos últimos anos devido ao aumento da expectativa de vida e modernização das tecnologias. De acordo com o Ministério da Saúde, o Brasil possui a quinta maior população idosa do mundo, com cerca de 28 milhões de pessoas com 60 anos ou mais (Brasil, 2016). É estimado que até 2025 a população idosa alcance $70 \%$ do total de pessoas no mundo, chegando em cerca de 840 milhões de idosos (World Health Organization, 2005).

Com esse aumento significativo a estimativa de crescimento chega a $223 \%$, e quando comparado as populações entre 1970 e 2025, as enfermidades e incapacidades físicas, cognitivas e emocionais também vão sofrer um avanço, fazendo com que haja maior necessidade de cuidadores contínuos (World Health Organization, 2005).

Nesse contexto, a profissão de cuidador de idoso vem ganhado destaque e importância no âmbito nacional. De acordo com o Ministério da Saúde, a ocupação de cuidador integra a Classificação Brasileira de Ocupações - CBO sob o código 5162, definindo assim o cuidador como alguém que "cuida a partir dos objetivos estabelecidos por instituições especializadas ou responsáveis diretos, zelando pelo bem-estar, saúde, alimentação, higiene pessoal, educação, cultura, recreação e lazer da pessoa assistida" (Brasil, 2008).

É a pessoa, da família ou da comunidade, que presta cuidados à outra pessoa de qualquer idade, que esteja necessitando de cuidados por estar acamada, com limitações físicas ou mentais, com ou sem remuneração (BRASIL, 2008).

A função do cuidador, seja ele um familiar ou da comunidade, é de auxiliar em atividades da vida diária de autocuidado, desde as mais básicas às mais complexas, dependendo da necessidade do idoso. Em uma análise da Fundação Oswaldo Cruz, com dados da Pesquisa Nacional de Saúde (2013), foi observado que 80\% dos idosos necessita de ajuda de algum familiar ou da comunidade para realizar atividades de vida diária, como vestir a roupa ou fazer compras (Brasil, 2016).

Diante desses fatores, destaca-se que, quanto mais dependente o idoso, maior será a sobrecarga sofrida ao cuidador, e assim, menor é a qualidade de vida deste com o passar do tempo. (Diniz, Lima \& Silva, 2017). Essa diminuição da qualidade de vida pode apresentar-se como uma exaustão emocional e mental devido ao envolvimento afetivo, com sentimento de incapacidade ao não conseguir uma melhora na qualidade de vida do idoso, em que este pode vir a óbito pela história natural da doença, desencadeando no cuidador quadros psiquiátricos de depressão e ansiedade. Outra forma de sobrecarga pode vir como prejuízo físico através de lombalgias, hipertensão arterial sistêmica e/ou outras comorbidades. 
Outro problema com elevada importância é que a maioria desses cuidadores possui a mesma faixa etária das pessoas que estão cuidando. Em um estudo feito por Karsch (2003), ele cita que 39.3\% de cuidadores entre 60 e 80 anos cuidavam de $62,5 \%$ de pacientes da mesma faixa etária, fazendo com que ele, uma pessoa que já possui fatores de risco para muitas enfermidades, tenha mais riscos a sua saúde.

Rosset (2021) constatou o predomínio do sexo feminino em cuidadores de idosos e divorciadas, assim gerando uma sobre carga devido á falta do suporte social e vê-se sozinhos nos cuidados gerando sobrecarga. O aumento de sobrecarga relaciona-se com aspectos da saúde física e mental, aumentando a probabilidade de doenças crônicas.

Portanto, este estudo justifica-se por dois motivos: a crescente preocupação com a qualidade de vida do cuidador, que muitas vezes é esquecida diante da gravidada da saúde do idoso incapacitado e a orientação aos cuidadores para que não negligencie sua saúde mental e física.

O objetivo principal deste artigo é analisar as evidências existentes na literatura sobre a qualidade de vida dos cuidadores de idosos, identificando as doenças mais prevalentes entre eles e a faixa etária.

\section{Metodologia}

Esse trabalho foi elaborado a partir de uma revisão de literatura por meio de artigos cientificos através de uma busca nas seguintes bases de dados: Lilacs, Google Acadêmico, Scientific Electronic Library Online - SciELO, US National Library of Medicine Nacional Institutes of Health (PubMed), BDNEF, Index Psicologia e Coleciona SUS, no período entre 2010 a 2021. Foram excluídos os artigos repetidos, que não se encaixavam no tema e que estavam fora do período descrito. A trajetória metodológica deste estudo sustentou-se nas análises de conteúdo e de citações do Ministério da Saúde (2008; 2015; 2016) e Organização Pan-Americana Da Saúde (2005), como suporte para a pesquisa.

Somando-se todas as bases de dados, foram encontrados 163 artigos. Os critérios de inclusão foram artigos originais e estudos transversais em que abordavam sobre a qualidade de vida de cuidadores de idosos, as principais patologias encontradas nessa população e as consequências que podem gerar a longo prazo. Selecionou-se inicialmente 20 artigos, porém após uma leitura detalhada de todos os estudos, os critérios de inclusão e exclusão possibilitaram a retirada de 2 artigos científicos. Diante disso, empregou 18 artigos para a análise do resumo. Foram usadas as seguintes palavras-chave e suas combinações, em inglês e português: cuidadores; envelhecimento; qualidade de vida; saúde do idoso.

\section{Resultados e Discussão}

Foram encontrados inicialmente 163 artigos. Após retirada dos artigos repetidos e que não atendiam os critérios de inclusão, restaram 18 estudos incluídos na revisão. Dentro dos artigos estudados, 3 pesquisaram a respeito da qualidade de vida dos cuidadores de idosos de forma abrangente, 1 relacionou a qualidade de vida com o índice de depressão, 9 estudos analisaram cuidadores de idosos com patologias como a Doença de Alzheimer (DA), Acidente Vascular Cerebral (AVC), demência, entre outras incapacidades funcionais. Também, 5 estudos relacionaram a qualidade de vida destes ao nível de sobrecarga decorrente dos afazeres diários.

Nardi, Sawada e Santos (2013) analisaram 178 idosos que apresentavam incapacidade funcional e seus respectivos cuidadores. Dentre os achados, em relação aos cuidadores familiares, 90,4\% eram do sexo feminino com média de idade de 56,7 anos e 45,4\% tinham idade acima de 60 anos. Ao analisar a idade do cuidador em relação a idade do idoso, constatou-se que cuidadores mais velhos cuidam em maior proporção de idosos mais velhos. Em relação a sobrecarga, foi identificado que 102 (57,3\%) apresentavam sobrecarga moderada; 44 (24,7\%) pequena sobrecarga; 28 (15,7\%) moderada a severa e apenas $4(2,2 \%)$ cuidadores com sobrecarga severa (Gráfico 1). Diante do proposto, identifica-se uma preocupação com os cuidadores de idosos que possuem idade acima de 50 anos, devido ao fato que, esses indivíduos possuem fatores de 
risco pré-existentes que podem aumentar devido à sobrecarga exacerbada.

Além disso, quando relacionado a dependência do idoso em relação a sobrecarga do cuidador, aqueles assistindo idosos classificados como independentes, apresentavam menores sobrecargas quando comparados a cuidadores que cuidam de idosos com dependência completa.

Gráfico 1 - Sobrecarga de cuidadores de idosos.

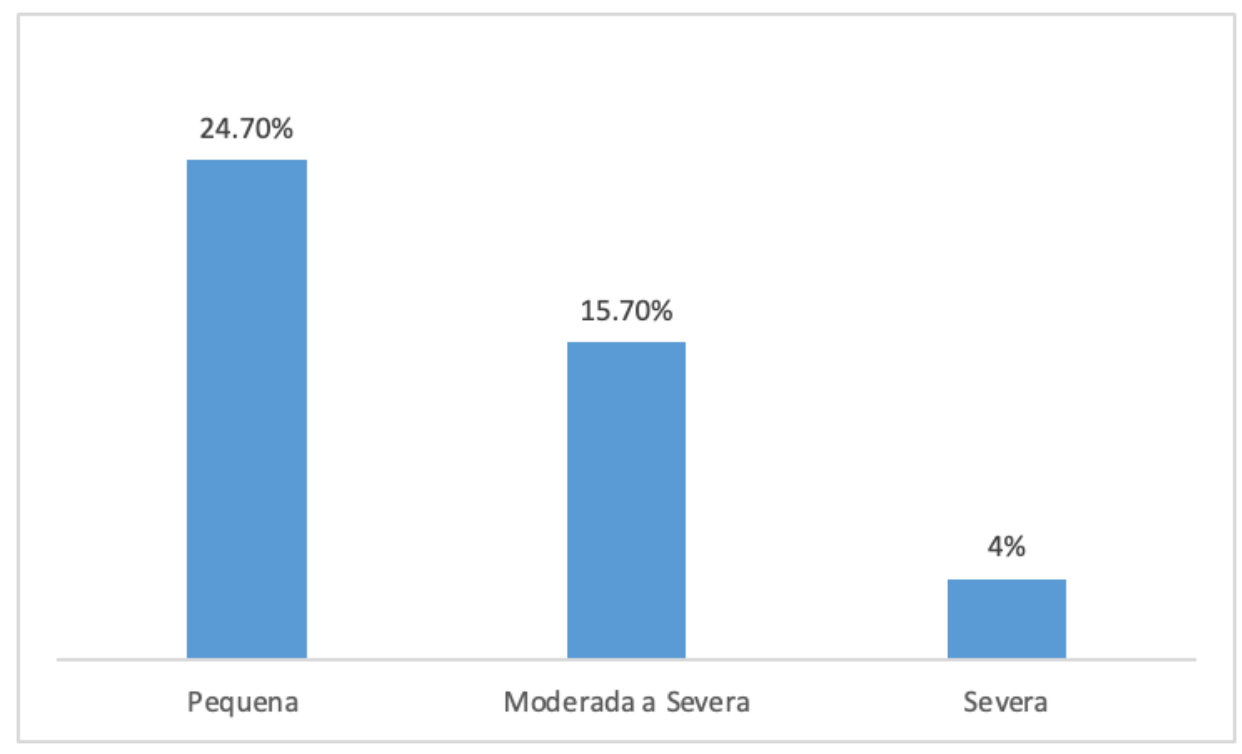

Fonte: Nardini, Sawada e Santos (2013).

Rondoni (2011) também analisou o nível de sobrecarga dos cuidadores. Foi, portanto, observado que quase metade das participantes, 82 (49,7\%), se mostraram insatisfeitas com o tempo para cuidar de si própria, devido o tempo empreendido no cuidar; 53 (32,3\%) afirmam que sua saúde foi afetada por causa do envolvimento com a pessoa cuidada; 86 $(53,3 \%)$ se dizem estressadas por cuidar dos idosos e ter suas outras responsabilidades com a família; 41 (24,8\%) mostraram preocupação em se tornar incapazes de continuar o cuidado por muito tempo; $39(23,8 \%)$ delas afirmaram sentir que perderam o controle da própria vida por conta do trabalho e $35(21,2 \%)$ declararam que gostariam de simplesmente deixar que outra pessoa cuidasse do idoso (Gráfico 2). Igualmente, mostra-se as principais preocupações desses cuidadores, que não apenas ocorrem com o cuidado próprio, como também o pressentimento de incapacidade de realizar a tarefa. Fatores dos quais podem ser precipitantes para doenças cardiovasculares e psiquiátricas devido ao estresse constante exercida pela sobrecarga do trabalho. 
Gráfico 2 - Principais preocupações dos cuidadores de idosos.

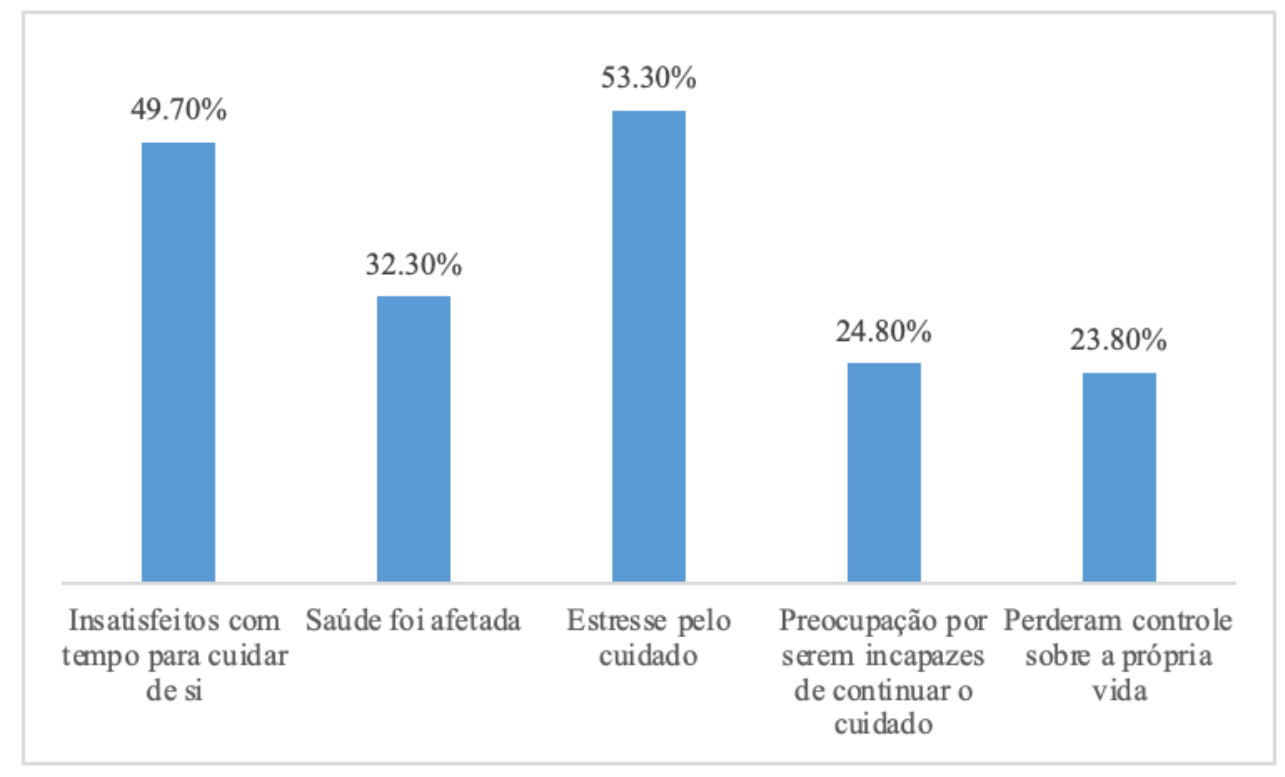

Fonte: Rondoni et. al., (2011).

Santos e Gutierrez (2013) e Manzini e Vale (2020) em ambos os estudos foram analisados cuidadores de idosos portadores de DA, sendo entrevistados 25 e 66 cuidadores, respectivamente, relacionando com a prevalência de doenças psiquiátricas e nível de estresse observado entre eles. Dessa forma, Santos e Gutierrez (2013) notou que $80 \%$ dos adultos e $100 \%$ dos idosos apresentavam médio traço de ansiedade e $72 \%$ dos adultos e $80 \%$ dos idosos apresentavam médio estado de ansiedade (Gráfico 3). Também, em relação a avaliação respondida pelos participantes em relação a satisfação com a saúde, constatou-se que 28 (56\%) deles estavam insatisfeitos com a sua saúde e 9 (18\%) estavam insatisfeitos. Já o estudo realizado por Manzini e Vale (2020) aqueles que cuidavam de idosos com a Doença de Alzheimer avançada, 47,3\% foram avaliados com sobrecarga intensa; $86,4 \%$ apresentaram níveis de estresse significativo; $57 \%$ apresentaram níveis graves de ansiedade e $36,9 \%$ apresentaram sintomas de depressão leve (Gráfico 4).

Tais fatores demonstram que os cuidadores possuem maiores probabilidades de apresentar problemas psiquiátricos, como a ansiedade, devido à grande responsabilidade que a tarefa de cuidar pode trazer, podendo afetar também na qualidade do sono, capacidade de atenção, tensão muscular, entre outros (Associação Brasileira de Psiquiatria, 2008).

Também, Cesário (2017) ao entrevistar 43 cuidadores, através do Inventário de Sintomas de Estresse para Adultos de Lipp (ISSL) e a Versão Brasileira do Questionário de Qualidade de Vida Short-form Health Survey (SF-36) concluiu que $27(62,8 \%)$ apresentam esse quadro, dentre eles $22(51,2 \%)$ estavam na fase de resistência e 5 (11,6\%) estavam quase na fase de exaustão (Gráfico 5). Em suma, a fase de resistência é caracterizada por um mal-estar generalizado, cansaço constante, tontura, aparecimento de problemas de pele. Já na fase de exaustão pode surgir diversos comprometimentos físicos em forma de doenças, como por exemplo tontura frequente, insônia, tiques nervosos, mudança extrema de apetite, irritabilidade, entre outros (Brasil, 2012). Consequentemente, esses cuidadores estão cada vez mais predispostos a sofrerem problemas de saúde progressivamente mais sérios, podendo chegar por exemplo a sofrer um infarto agudo do miocárdio (IAM) e morte súbita (Lee et al., 2003). 
Research, Society and Development, v. 10, n. 15, e282101523024, 2021

(CC BY 4.0) | ISSN 2525-3409 | DOI: http://dx.doi.org/10.33448/rsd-v10i15.23024

Gráfico 3 - Relação entre traço e estado de ansiedade entre idosos e cuidadores.

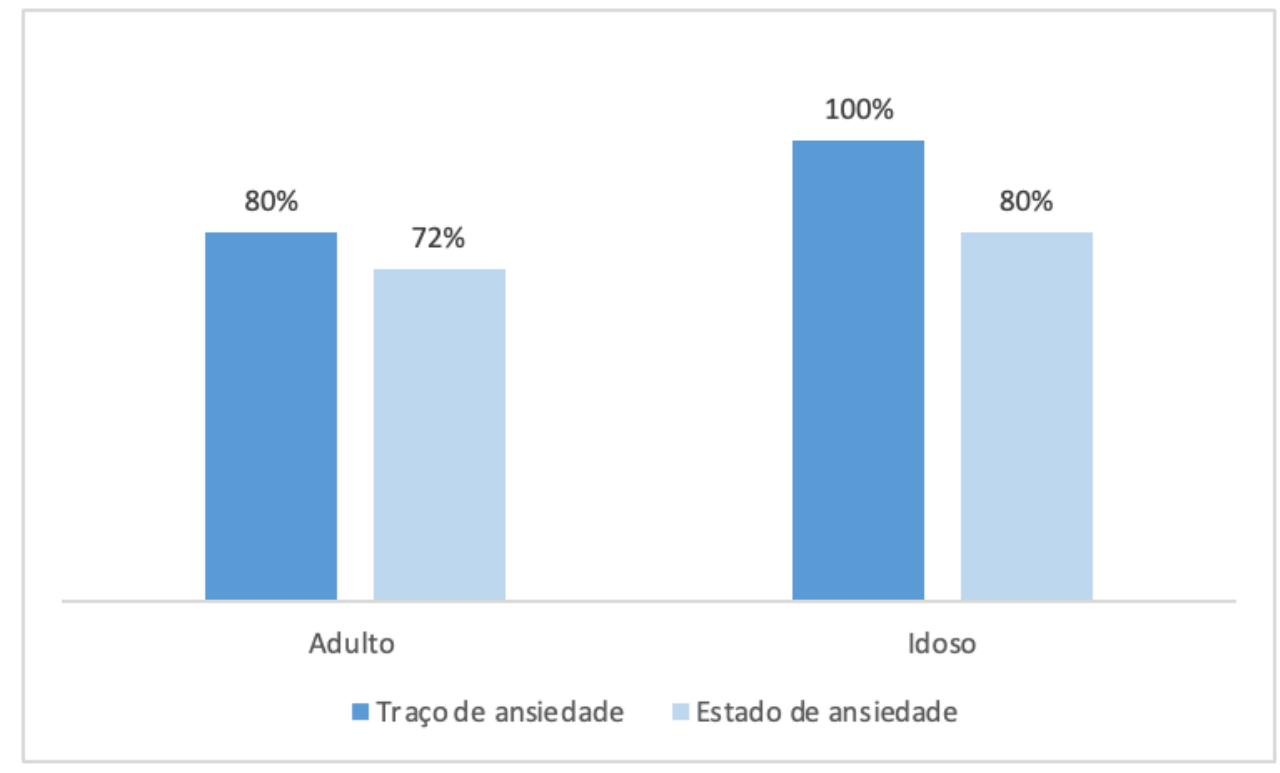

Fonte: Santos e Gutierrez (2013).

Gráfico 4 - Sintomas avaliados nos cuidadores de idosos com DA.

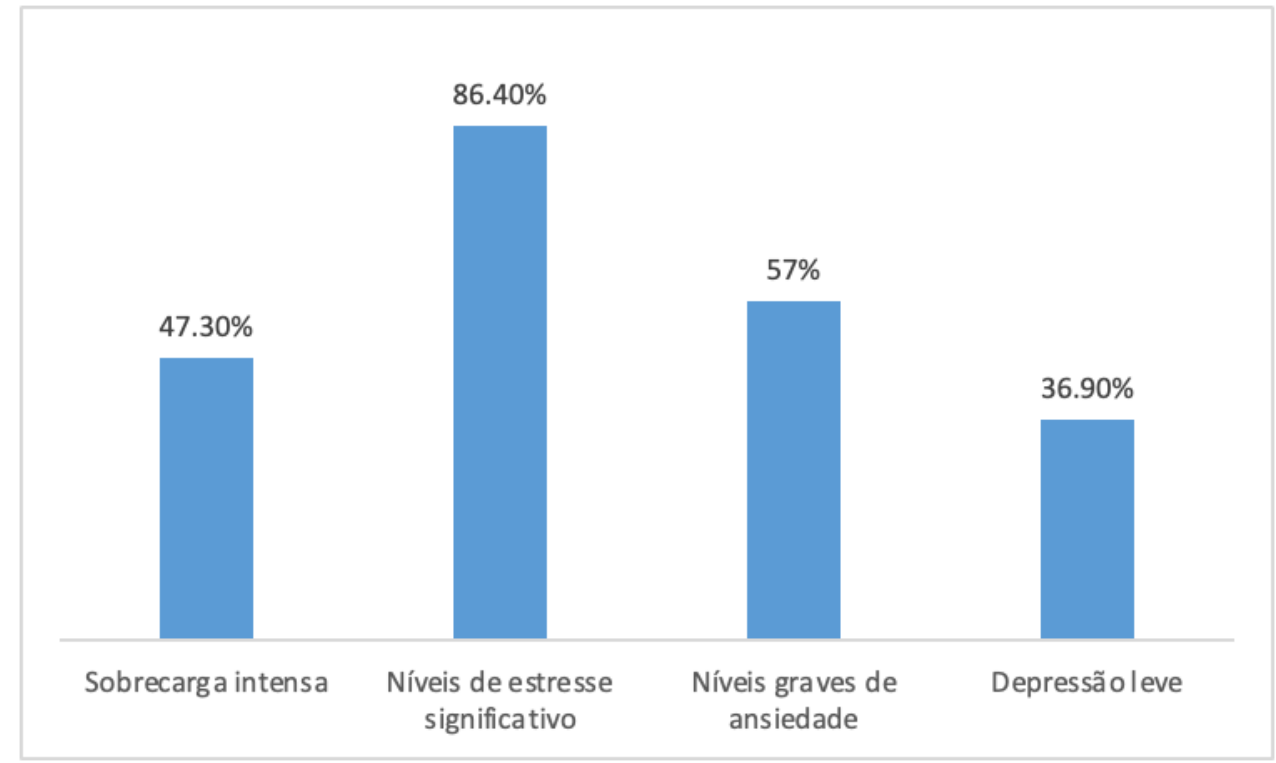

Fonte: Manzini e Vale (2020). 
Gráfico 5 - Nível de estresse dos cuidadores de idosos.



Fonte: Cesário et. al., (2017).

Quando analisado os cuidadores de idosos com demência, Dias (2018) analisou a sobrecarga devido aos cuidados de 31 cuidadores informais. Por conseguinte, ao serem questionados sobre a sobrecarga, 14 (45,17\%) não relataram sobrecarga, $8(25,81 \%)$ sobrecarga moderada e $9(29,04 \%)$ sobrecarga severa (Gráfico 6).

Gráfico 6 - Sobrecarga dos cuidadores de idosos diagnosticados com demência.

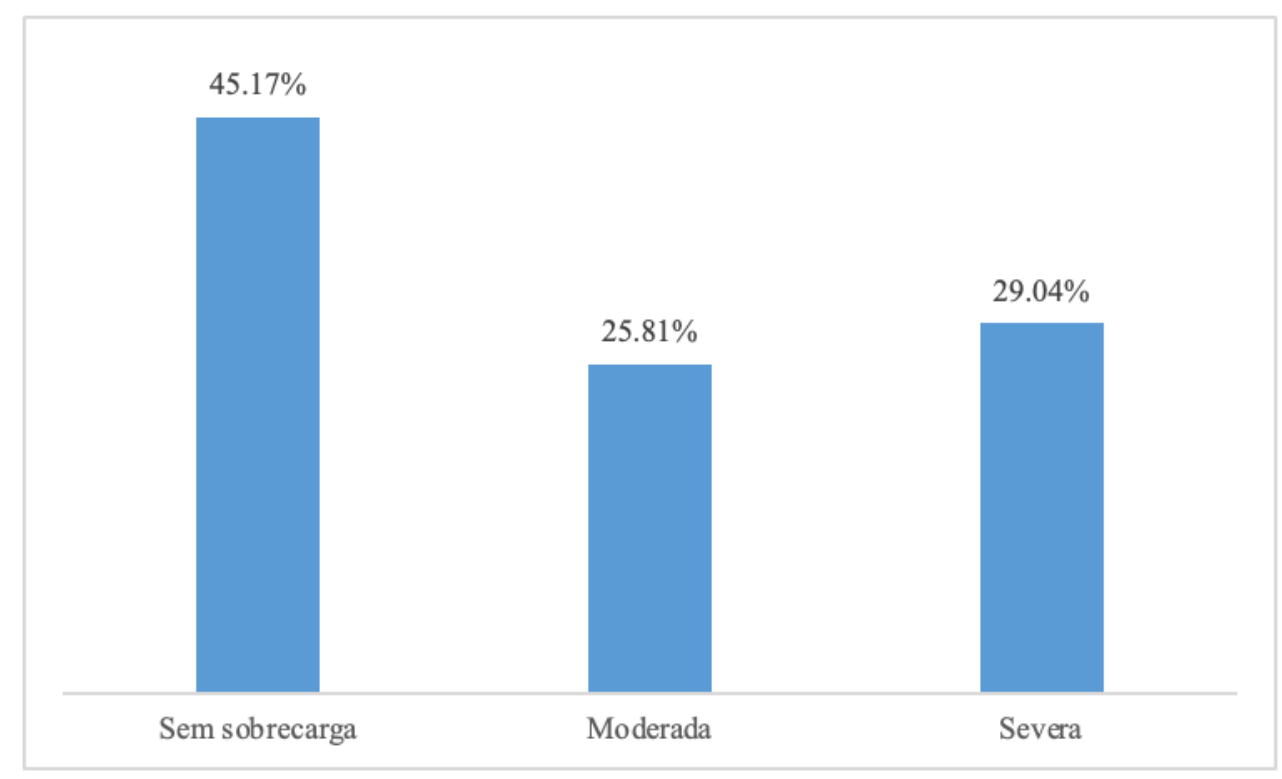

Fonte: Dias (2018).

Ainda no âmbito da sobrecarga exercida pelo cuidador, Oliveira (2018) realizou um estudo em que foram entrevistados 75 cuidadores, pelos quais 81,3\% correspondiam ao sexo feminino, com idade média de 69,8 anos. A entrevista foi realizada através dos testes qui-quadrado, teste U de Mann-Whitney e Kruskal-Wal. Em vista disso, nota-se que os idosos cuidadores com níveis mais altos de sobrecarga $(\geq 29)$ tem 11,4 vezes mais chance de pontuar para pior qualidade de vida.

Segundo Santos e Tavares (2011), através de um estudo tipo inquérito domiciliar ao avaliar a qualidade de vida os cuidadores a consideravam boa $(50 \%)$; nem ruim nem boa $(37 \%)$; muito ruim $(6,5 \%)$ e muito boa $(6,5 \%)$. Quanto à 
autoavaliação dos cuidadores em relação à satisfação com a sua saúde, 41,2\% disseram estar satisfeitos, 19,6\% insatisfeitos, 19,6\% muito satisfeitos, $13 \%$ nem satisfeitos, nem insatisfeitos e 6,5\% muito insatisfeitos (Gráfico 7). Além disso, foi notada uma relação negativa entre o número de morbidades e os escores de QV nos domínios físicos e psicológicos, sendo que, quanto maior o número de morbidades do cuidador, menor escore de QV em ambos domínios.

Diante do proposto, demonstra-se que muitas vezes o cuidador é impedido de cuidar da sua própria saúde, devido à sobrecarga e falta de tempo disponível, e assim, desenvolve sentimentos negativos em relação a sua imagem corporal. Isso não traz malefícios apenas para ele, mas também ao idoso, já que o cuidador pode criar um bloqueio de vínculo e começa a negar cuidado de qualidade e carinho ao idoso. Portando, é necessário incentivar o cuidador a valorizar suas habilidades e sentimento para reverter tal quadro (Santos \& Tavares, 2011).

Gráfico 7 - Qualidade de vida e Satisfação com a saúde dos cuidadores de idosos.

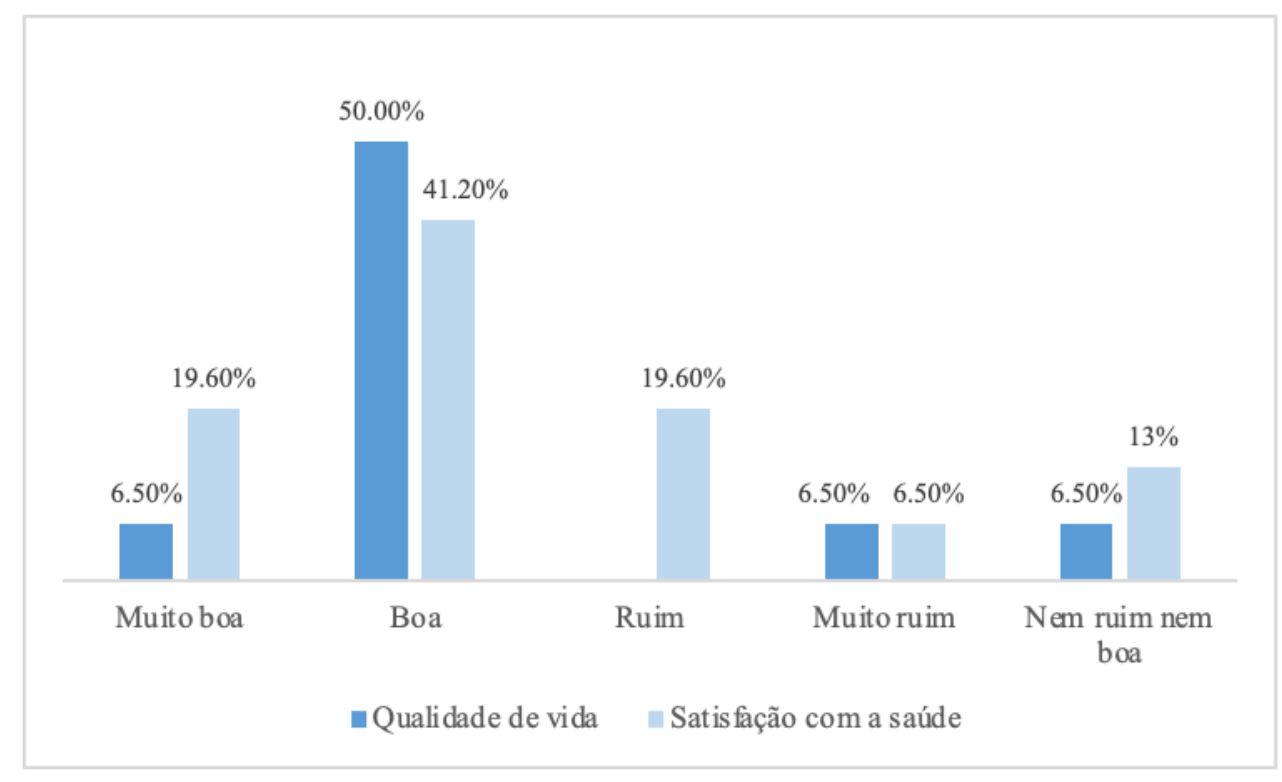

Fonte: Santos e Tavares (2011).

Queiroz (2018), Maciel (2015) e Cedano (2013) observaram nos seus estudos algumas comorbidades recorrentes entre os cuidadores de idosos, sendo entrevistados 35, 31 e 80 cuidadores, respectivamente. Dentre as comorbidades foram analisadas a prevalência de doenças cardiovasculares, como a hipertensão arterial sistêmica (HAS), diabetes mellitus (DM), depressão e doenças musculoesqueléticas, como lombalgia, artrose, atrite, escoliose, cifose, entre outras. Por conseguinte, Queiroz (2018) notou que 85,7\% deles apresentavam algum problema crônico de saúde, sendo as enfermidades mais frequentes: problemas de coluna $(33,3 \%), \operatorname{HAS}(28,2 \%), \mathrm{DM}(12,8 \%)$ e depressão $(7,7 \%)$ (Gráfico 8). A respeito da autoavaliação da qualidade de vida foi obtido um predomínio na resposta "nem ruim nem boa" com 48,6\%. No domínio psicologia a questão pior avaliada foi com relação a sentimentos negativos, onde $16(45,7 \%)$ dos participantes relataram que algumas vezes possuíam mau humor, desespero, ansiedade e depressão, e 7 (20\%) aprontaram que frequentemente possuíam esses sentimentos.

Esses aspectos podem ser explicados porque, geralmente, no início da doença existe uma maior integração entre familiares e amigos do idoso. Entretanto, com a evolução da doença, isto tende a mudar, e assim, o confinamento domiciliar junto com a falta de lazer leva a uma maior sobrecarga do cuidador, afetando sua capacidade, isolamento, alteração no estilo de vida e insatisfação pessoal (Pereira \& Oliveira 2012).

Maciel (2015) em seu estudo constatou que 60\% deles referiram ter algum problema de saúde, sendo mais 
frequente as dores articulares e musculares (16,13\%) diabetes mellitus (12,90\%) e hipertensão arterial (12,90\%) (Gráfico 8). Entre eles, 73,33\% realizavam consultas médicas regularmente, 66,67\% nunca se consultaram com uma nutricionista, $83,87 \%$ não praticavam exercícios físicos regulares. Também, foi destacado que $67,74 \%$ dos cuidadores apresentavam risco para o desenvolvimento de doenças cardiovasculares de acordo com a medida da circunferência da cintura.

Cadeno (2013) também notou que a maioria dos cuidadores eram portadores de alguma morbidade com prevalência de distúrbios cardiovasculares (65\%), sendo elas: hipertensão arterial, infarto agudo do miocárdio e trombose; e doenças musculoesqueléticos (25) sendo elas: artrite, artrose, escoliose, cifose, osteoporose e hérnia de disco. Também, observou-se que $8(10 \%)$ apresentavam depressão.

Anastácio (2021) traz em seu estudo algo que pode influenciar positivamente na qualidade de vida dos trabalhadores, sendo este o prazer. Sendo, diante disso, a troca de vínculos de ambos, do cuidador e do idoso, como uma fonte de alegria, gerando uma maior satisfação diante ao reconhecimento pelo seu trabalho ao exercer tal ocupação. Assim, atribuindo sentimento de orgulho e utilidade quando relatam a sua contribuição para o alivio da dor e avanço do estado de saúde dos idoso.

Gráfico 8 - Comorbidades encontradas entre cuidadores de idosos.

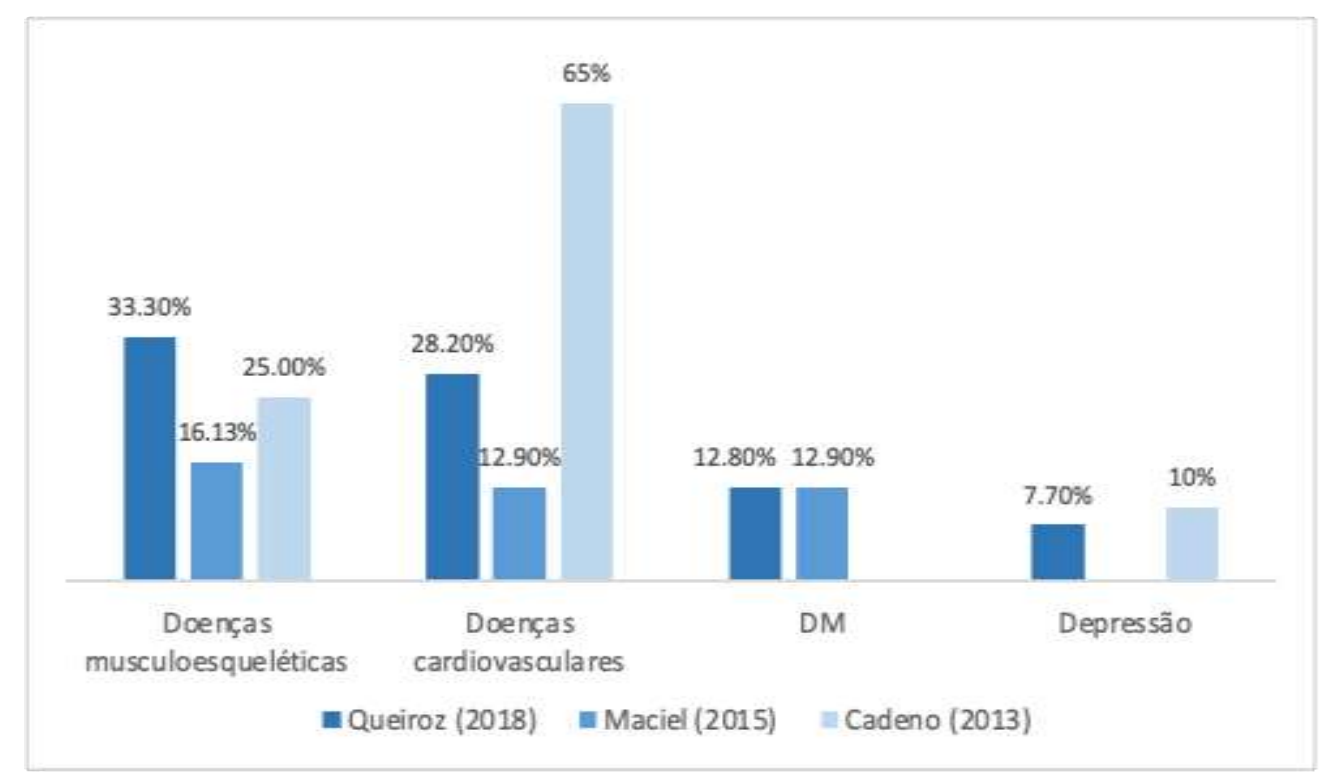

Fonte: Queiroz (2018); Maciel (2015); Cadeno (2013).

Reis, Dourado e Guerra (2019) também descreveram sobre as doenças cardiovasculares e seus fatores de risco em um estudo com 34 cuidadoras de idosos. Desta forma, notou-se que $80 \%$ da amostra encontrava-se com sobrepeso ou obesidade, $33 \%$ com HAS, $85 \%$ com a circunferência da cintura elevada ou muito elevada, $62 \%$ com circunferência do pescoço aumentada e 74\% com razão cintura/quadril aumentada (Gráfico 9).

Anjos, Boery e Pereira (2015) realizaram um estudo com 58 cuidadores de idosos no interior do estado da Bahia, e diante disso, observaram as comorbidades mais prevalentes naquele grupo. Por conseguinte, em relação as doenças referidas pelos cuidadores, verificou-se a predominância do diagnóstico de lombalgia (63,8\%), seguido de hipertensão arterial sistêmica $(41,4 \%)$ e varizes $(37,9 \%)$ (Gráfico 10$)$.

Diante disso, mostra-se a tendência para efeitos negativos relacionados ao ato de cuidar, com destaque a doenças físicas, psicossomáticas, ansiedade e depressão. Porém, não são todos os cuidadores que desenvolvem essas doenças ou se sentem insatisfeitos com a tarefa de cuidar. Tal fato pode ser explicado por estratégias individuais para lidar com as situações 
desgastantes do dia a dia (Gaioli, Furegato \& Santos, 2012).

Gráfico 9- Comorbidades e fatores de risco encontradas entre cuidadores de idosos.



Fonte: Reis, Dourado e Guerra (2019).

Gráfico 10- Comorbidades mais prevalentes entre os cuidadores no interior do estado da Bahia.

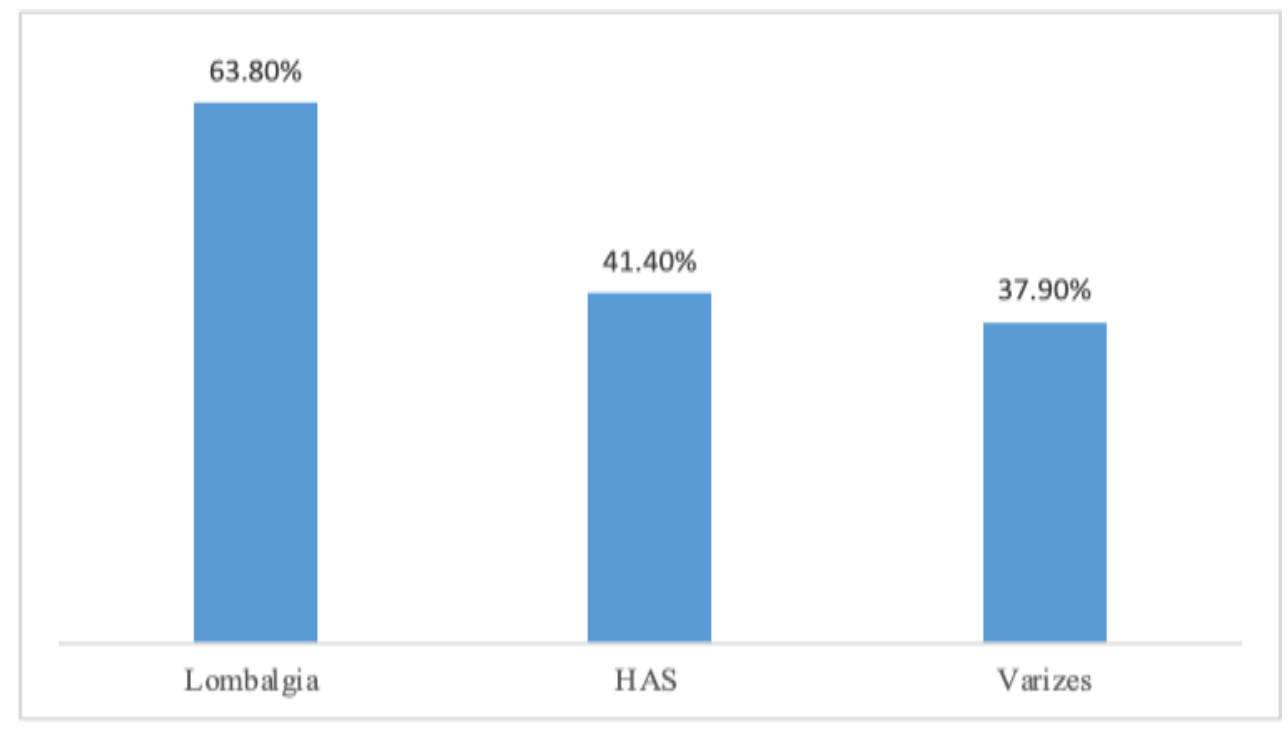

Fonte: Anjos, Boery e Pereira (2015).

No âmbito de doenças psiquiátricas, Sampaio (2018) através de seu estudo entrevistou 35 cuidadores de idosos para observar a qualidade de vida deles (Gráfico 11). Dessa maneira, 50\% dos cuidadores apresentavam indicativo de depressão, dos quais, 11 (30,6\%) com depressão leve, 11,1\% depressão moderada e 8,3\% depressão severa (Gráfico 10). Em relação a qualidade de vida, $83,66 \%$ referiram como média e $60,88 \%$ referiram sentir dores. A literatura afirma que o cuidador de idosos, quando comparado a um indivíduo não cuidador, está mais vulnerável a adquirir determinadas doenças, com destaque na depressão (Pinto \& Barham, 2014). Sintomas depressivos podem ser caracterizados como: tristeza, choro, desprazer, diminuição da autoconfiança, irritação, desanimo, dificuldade de relacionar-se com outras pessoas (Associação Brasileira de Psiquiatria, 2001). 
Gráfico 11- Classificação da depressão entre os cuidadores de idosos.

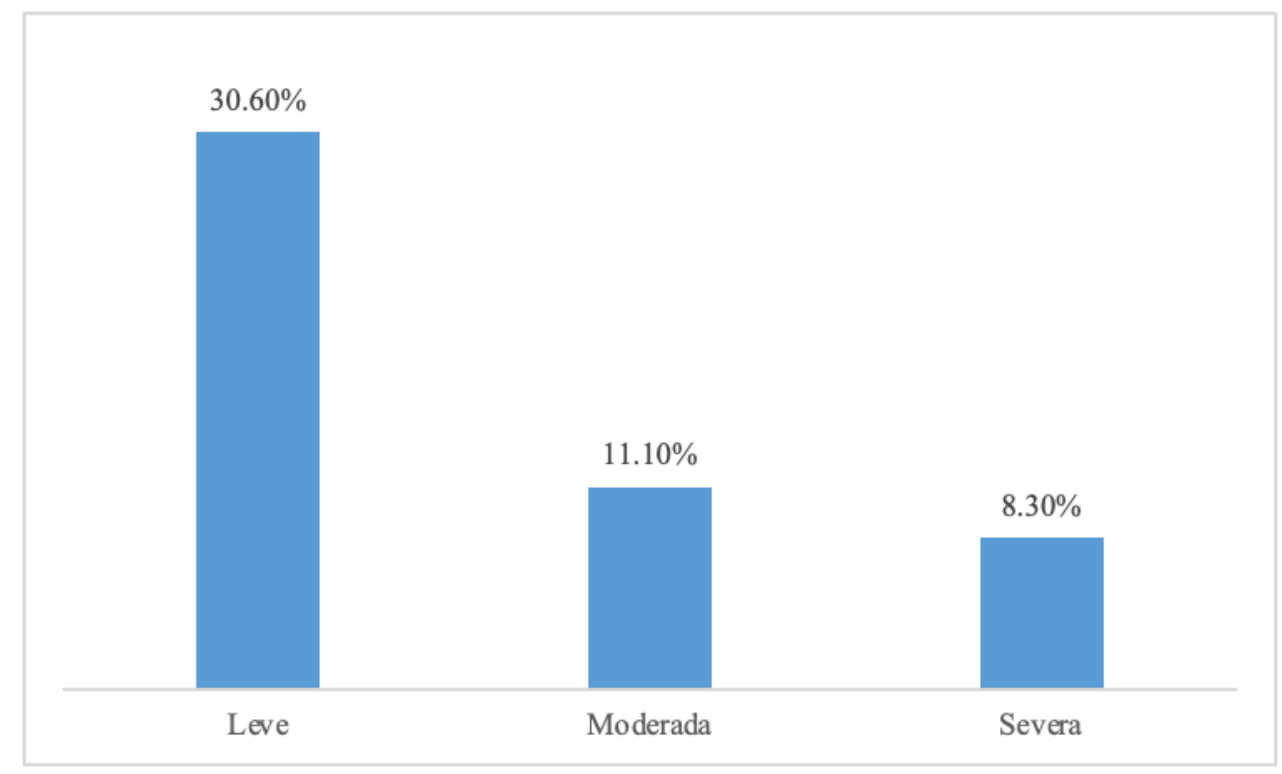

Fonte: Sampaio (2018).

Lago (2015) em seu estudo destacou no seu estudo não apenas as comorbidades mais prevalentes, como também em relação ao uso de medicações como ansiolíticos e antidepressivos utilizados por essa população. Diante dos 33 participantes, percebe-se que as maiores queixas dos entrevistados eram de dores na coluna (45\%), membros e articulação (39\%) (Gráfico 12). A maioria faz usos de medicamentos para aliviar a dor ou para a indução do sono constantemente, ou fazem uso de ansiolíticos (24\%), antidepressivos (18\%). O sono avaliado em qualidade e quantidade teve um resultado ruim, sendo que a maioria dos participantes revelou ter menos de 6 horas de sono diária. Por conseguinte, Lago (2015) cita em seu estudo que cuidar de alguém de forma ininterrupta pode representar altos custos no nível psíquico, físico e social. Além disso, o predomínio das doenças osteoarticulares mostra o grande desgaste físico que é percebido nesses cuidadores devido a movimentação necessária para o exercício do cuidado, principalmente em pacientes acamados ou com algum grau importante de limitação física. Também é importante destacar que, muito desses cuidadores não realizam atividade física ou exercícios de fortalecimento, e diante disso, favorece o surgimento de lesões em articulações e dores musculares por esforço.

Em consequente ao exposto, muito desses cuidadores utilizam medicamentos para alívio das dores, muitas vezes sem prescrição médica e de medicamentos antidepressivos e ansiolíticos prescritos por médicos se tornou frequente, inclusive para induzir o sono, sendo que a dificuldade para dormir também pode ser consequência da dor (Lago et al., 2015). 
Gráfico 12 - Patologias e uso de medicações mais prevalentes entre os cuidadores de idosos.



Fonte: Lago (2015).

O estudo realizado por Pereira e Carvalho (2012) revelou que a qualidade de vida dos 92 cuidadores entrevistados, estava negativamente relacionada com a sobrecarga e morbidade e positivamente com a satisfação com os amigos. A sobrecarga e qualidade de vida correlacionaram-se com o suporta social total. O ajustamento conjugal moderou a relação entre depressão e qualidade de vida (dimensões física e psicológica), mas não entre sobrecarga e qualidade de vida. Igualmente, os cuidadores cônjuges apresentam menor qualidade de vida e os cuidadores filhos, mais morbidade psicológica. Verificou-se também que cuidadores que possuem apoio ao nível da prestação de cuidados apresentam maior satisfação conjugal. Os resultados, portanto, enfatizam a importância de intervir junto desta população no sentido de aumentar a qualidade de vida dos cuidadores.

Barbosa (2017) fez um estudo com 92 cuidadores, sendo constituído a maioria por mulheres $(84,8)$ com a faixa etária acima de 30 anos (73\%). Quando analisada a qualidade de vida desses cuidadores, 16 (17,4\%) relataram que acredita estar um pouco pior/muito pior quando comparado há um ano atrás. Percebeu-se também que ocorreu uma maior deterioração da saúde para aqueles que estão na ocupação há cinco anos ou mais, e para aqueles que exerciam a ocupação há 10 anos ou mais, constatou-se diferença significativa no domínio da capacidade funcional. Em conclusão, nota-se que o tempo de cuidado pode interferir positivamente, devido ao vínculo criado entre o idoso e o cuidador, ou de forma negativa, tendo que entrar em questão as condições sociodemográficas inclusas e o grau de dependência do idoso.

\section{Conclusão}

Identifica-se que com o aumento da expectativa de vida, houve consequentemente um aumento de profissionais da área de cuidadores de idosos, dando um novo olhar a estes trabalhadores. Diante do presente estudo, com análise dos resultados e da discussão desta revisão, conclui-se que vários fatores influenciam para a qualidade de vida desses trabalhadores, seja de forma positiva ou negativa. Tais fatores variam entre a sobrecarga e ao vínculo criado entre cuidador e o idoso, gerando assim, maior predisposição a doenças cardiovasculares e psiquiátricas, como depressão e ansiedade principalmente devido ao estresse e sobrecarga emocional, como também hipertensão, doenças musculoesqueléticas como artrose, lombalgia, cifose, entre outras, principalmente em razão do esforço físico repetitivo.

Analisa-se, a partir dos resultados, que diversos fatores influenciam diretamente para o aumento das 
comorbidades citadas, dentre elas se destacam o grau de dependência do idoso, sobrecarga exercida, grau de esforço, má qualidade do sono, condições financeiras, saúde e faixa etária do cuidador, visto que muitos cuidadores estão cuidando de idosos com idade semelhante. Alguns outros fatores podem exercer efeito aditivo na sobrecarga, e assim na percepção da qualidade de vida, são elas então, as situações de estresse, desgaste e cansaço que também podem influenciar.

Nota-se, então, a necessidade de sistematizar estratégias de ajuda a esses trabalhadores, com novos estudos que ofereça estratégias de intervenção baseadas em evidência que possa ajuda-los na prática. Assim, deixando-a mais simples, prazerosa e humanizada, voltada para aspectos psicossociais com o cuidado e ao convívio com o idoso dependente de cuidados, afim de evitar agravos destes cuidadores.

Por conseguinte, fica claro a necessidade do olhar mais cuidadoso perante a qualidade de vida dos cuidadores de idosos devido ao prejuízo à saúde destes, seja física ou emocional. Cabe, então, à equipe de saúde a realização de estratégias de intervenção, criando vínculos terapêuticos com as famílias dos idosos e aos cuidadores, identificando as suas necessidades, a fim de diminuir o estresse e sobrecarga, organizando e sistematizando o cuidado, também, com planos de lazer ao ar livre, gerando maior prazer e distração de ambas as partes. Com ênfase na relação dentre os cuidadores, criando uma maior rotatividade de cuidado e assim, um maior tempo de lazer a eles, para que haja aperfeiçoamento do tempo de autocuidado. Igualmente, precisa-se maior suporte social e acolhimento a suas queixas, que geralmente sofrem com o descaso e esquecimento dos governantes. Também, é necessário a criação de políticas de saúde para prevenir e tratar doenças características dessa população, além do mais, leis que possibilitam uma remuneração salarial digna diante de longas horas de jornada de trabalho. Sendo assim, é enfatizado como estratégias de melhoria de qualidade de vida suporte educacional, político, social, financeiro e psicológico, dando um olhar mais humanizado a vida desses trabalhadores formais e informais.

\section{Referências}

Anastácio, F., et al. (2021). Qualidade de vida de trabalhadores de instituição de longa permanência para idosos: revisão de escopo. Research, Society and Development, 10(14), e429101422022.

Associação Brasileira De Psiquiatria. (2001). Diagnóstico e Tratamento da Depressão. Associação Médica Brasileira e Conselho Federal de Medicina.

Associação Brasileira De Psiquiatria. (2008). Transtorno de Ansiedade: Diagnóstico e Tratamento. Associação Médica Brasileira e Conselho Federal de Medicina.

Anjos, K., Boery, R. \& Pereira, R. (2015). Qualidade de Vida de Cuidadores Familiares de Idosos Dependentes no Domicílio. Texto Contexto Enfermagem, Florianópolis, 23(3): 600-8.

Barbosa, L., et. al. (2017). Qualidade de vida relacionada à saúde dos cuidadores formais de idosos institucionalizados em Natal, Rio Grande do Norte. Revista Brasileira de Estudos de População, 34 (2): 391-414.

Brasil. Ministério da Saúde. (2008). Guia Prático do Cuidador. Brasília - DF.

Brasil. Ministério da Saúde. (2015). Estresse. Portal do Governo Brasileiro.

Brasil. Ministério da Saúde. (2016). Ministério recomenda: é preciso envelhecer com saúde.

Cedano, S., et.al. (2013). Qualidade de vida e sobrecarga dos cuidadores de portadores de Doença Pulmonar Obstrutiva Crônica em oxigenoterapia. Revista Latino-Americano de Enfermagem, 21(4): 860-7.

Cesário, V., et. al. (2017). Estresse e qualidade de vida do cuidador familiar de idoso portador da doença de Alzheimer. Saúde Debate, Rio de Janeiro, 4 (112): $171-182$.

Dias, L., et. al. (2018). Sobrecarga no cuidado de paciente idoso com demência. Revista Kairos - Gerontologia, 21 (1): 169-190.

Diniz, A., Lima, R. \& Silva, B. (2017). Sobrecarga do cuidador de idosos: uma revisão integrada. Revista Pesquisa de Saúde, 18 (3): $184-188$.

Gaioli, C., Furegato, A. \& Santos, J. (2012). Perfil de cuidadores de idosos com doença de Alzheimer associado à resiliência. Texto Contexto Enfermagem, vol. 21 (1): 150-7.

Karsh, U. (2003). Idosos dependentes: famílias e cuidadores. Caderno de Saúde Pública, Rio de Janeiro, 19 (3): $861-866$.

Lago, Diane et. al. (2015). Sobrecarga Física e Psicológica dos Cuidadores de Pacientes Internados em Domicílio. Revista Enfermagem UFPE online, Recife, 9(1): 319-26. 
Lee, S., et. al. (2003). Caregiving and risk of coronary heart disease in U.S. women: a prospective study. Am J Prev Med, 24(2): 113-9.

Maciel, A., et. al. (2015). Qualidade de vida e estado nutricional de cuidadores de idosos dependentes. Revista Kairós Gerontologia, 18 (4):179-196.

Manzini, C. \& Vale, F. (2020). Emotional disorders evidenced by family caregivers of older people with Alzheimer's disease. Dement Neurophycol, 14(1): 5661.

Nardi, E., Sawada, N. \& Santos, J. (2013). Associação entre a incapacidade funcional do idoso e a sobrecarga do cuidador familiar. Revista Latino Americano de Enfermagem, 21(5): 1096-103.

Oliveira, J., et. al. (2018). Qualidade de vida de idosos que cuidam de outros idosos com doenças neurológicas. Revista Brasileira de Geriatria e Gerontologia, Rio de Janeiro, 21(4): 440-451.

Organização Pan-Americana Da Saúde. (2005). Envelhecimento ativo: uma política de saúde. Brasília - DF.

Pereira, M. \& Carvalho, H. (2012). Qualidade vida, sobrecarga, suporte social, ajustamento conjugal e morbidade psicológica em cuidadores de idosos com dependência funcional. Temas em Psicologia, 20(2): 369-383.

Pereira, L. \& Oliveira, A. (2012). Cuidadores de idosos dependentes no domicilio: mudanças nas relações familiares. Revista Brasileira de Enfermagem, 65 (5): 730-6.

Pinto, F. \& Barham, E. (2014). Bem-estar psicológico: comparação entre cuidadores de idosos com e sem demência. Psicologia, Saúde e Doenças, 15(3): 635655 .

Queiroz, R., et. al. (2018). Perfil sociodemográfico e qualidade de vida de cuidadores de idosos com demência. Revista Brasileira de Geriatria e Gerontologia, Rio de Janeiro, 21(2): 205-214.

Reis, E., Dourado, V. \& Guerra, R. (2019). Qualidade de Vida e Fatores de Risco à Saúde de Cuidadoras Formais de Idosos. Estudo Interdisciplinar de Envelhecimento, Porto Alegre, 24(1): 47-61.

Rondini, C., et. al. (2011). Analise das relações entre qualidade de vida e sobrecarga de cuidadores de idosos de Assis, SP. Estudos e pesquisas em psicologia, Rio de Janeiro, 11(3): 796-820.

Rosset B., et al (2021). Qualidade de vida de cuidadores de idosos leigos associada ao perfil sociodemográfico e situação de saúde. Society and Development, 10(13): 1-9.

Sampaio, L., et.al. (2018). Qualidade de Vida e Depressão em Cuidadores de Idosos Dependentes. Revista APS, $21(1)$ : $112-121$.

Santos, C. \& Gutierrez, B. (2013). Avaliação da Qualidade de Vida de Cuidadores Informais de Idosos Portadores da Doença de Alzheimer. Revista Mineira de Enfermagem, 17(4): 792-798.

Santos, N. \& Tavares, D. (2012). Correlação entre qualidade de vida e morbidade do cuidador de idoso com acidente vascular encefálico. Revista da Escola de Enfermagem da USP, 46 (4): 960-6. 